\title{
The sovereignty of Artificial Intelligence over Human Ethics and Heedfulness
}

\author{
Sunil Varma Mudundi ${ }^{1}$, Tejaswi Pasumathy ${ }^{1}$ and Dr. Raul Villamarin Roudriguez ${ }^{2}$ \\ ${ }^{1}$ Post graduation scholar, School of Business, Woxsen University, Telangana, India. \\ ${ }^{2}$ Pro Vice Chancellor, Woxsen University, Telangana, India.
}

\begin{abstract}
:
Artificial Intelligence in present days is in extreme growth. We see AI in almost every field in work today. Artificial Intelligence is being introduced in crucial roles like recruiting, Law enforcement and in the Military. To be involved in such crucial roles, it needs lots of trusts and scientific evaluation. With the evolution of artificial intelligence, automatic machines are in a speed run in this decade. Developing a machine/robot with a set of tools/programs will technically sort of some of the challenges. But the problem arises when we completely depend on robots/machines. Artificial intelligence this fast-growing technology will be very helpful when we take help from it for just primary needs like face detection, sensor-controllers, bill counters...etc. But we face real challenges when we involve with decision making, critical thinking...etc. In mere future, automated machines are going to replace many positions of humans. Many firms from small to big are opting for Autonomous means just to make their work simpler and efficient. Using a machine gives more accurate results and outputs in simulated time. As technology is developing fast, they should be developed as per societal rules and conditions. Scientists and analysts predict that singularity in AI can be achieved by 2047. Ray Kurzweil, Director of Technology at Google predicted that AI may achieve singularity in 2047. We all saw the DRDO invention on autonomous fighting drones. They operate without any human assistance. They evaluate target type, its features and eliminate them based on edge detection techniques using computer vision. AI is also into recruiting people for companies. Some companies started using AI Recruiter to evaluate the big pool of applications and select efficient ones into the industry. This is possible through computer vision and machine learning algorithms. In recent times AI is being used as a suggestion tool for judgement too. Apart from all these advancements, some malicious scenarios may affect humankind. When AI is used in the wrong way many lives will fall in danger. Collecting all good and evil from past experiences is it possible to feed a machine to work autonomously. As many philosophers and educated people kept some set of guidelines in society is it practically possible to follow when AI achieves singularity and when we talk about the neural networking of human. They have good decision-making skills, critical thinking...etc. We will briefly discuss the ethics and AI robots / Machines that involve consciousness and cognitive abilities. In this upgrading technological world, AI is ruling a maximum number of operations. So, we will discuss how can ethics be followed. How can we balance ethics and technology in both phases. We will deep dive into some of these interesting areas in this article.
\end{abstract}




\section{Literature Review:}

Consciousness: This is a concept in philosophy, sociology, psychology. Consciousness is defined as a combination of both arousal and awareness. We all know arousal will be controlled by the brainstem. As we discussed awareness and wakefulness are two main components of consciousness. We can describe arousal as the level of consciousness. Whereas self-awareness can be explained in emotions, imagination, daydreams. These all are internal awareness. There is also external awareness. Here this can be experienced using the five senses of humans. Consciousness is evolved while vertebrates compete with invertebrates in the sea. Invertebrates, forebrain vesics forms a hollow tube. It specifies the presence of tectum. Tectum is nothing but the roof in "Latin". This covers the top of the brain. This allowed vertebrates to develop complex neural systems.

Artificial Intelligence: We had seen three revolutions that changed the path of the world. One was the "First steam engine" in the year 1784. The second was "Invention of Electricity" in the year 1969. When we talk about the third "Evolution of Information \& Technology". Now we are into the fourth phase of revolution "Artificial intelligence". In this phase, we see automation of machines. Simply it is a collection of programs where it makes a user feel like dealing with a human being. In the evolution of Artificial Intelligence, there are three phases namely

The first chatbot ELIZA was invented in 1964-1966. This comes under the ANI era of artificial intelligence. We are now in the phase of AGI. Robots thinking like humans making decisions without human intervention comes in the ASI phase of the Artificial Intelligence revolution.

Cognition:

This can be described as the process of acquiring knowledge and gathering information thought, senses, and experiences. The meaning of the word cognition is "People Think". When we read it in two CO- "Together" and GNOSCERE- "Know". There are mainly seven cognitive abilities namely Attention, Memory, Processing, Perception, Mobility, Logic \& Reasoning. These skills are very important to a human being to carry out tasks starting from simple to complex. Cognition is not a fancy word or an invention. It is mental processing that is present in every creature on earth from the first life on earth. Apes, monkeys memorize their children, sense their surroundings. Cognition is the most important skill humans have compared to other animals and living beings. Using cognition every individual can make decisions.

\section{Society with AI:}

Society is simply a group of people with the common region, culture and many else. Generally, when we talk in human terms about society it specifies that society is a group that consists of two or more people who have some interaction and identity with one another. For example, if we consider two different countries like Afghanistan and Pakistan. They both are geographically different, but they are in the same society following Islam as a basis. They both follow the same language as Urdu. So, when we discuss materialistic society. Artificial Intelligence and its applications can be called Materialistic Society ${ }^{[1]}$. As it has no birth or death as humans. In this society machines or robots will interact with other machines in work culture or a system. Machines can communicate with other machines to get work done at the right time. While considering this materialistic society scientists and research scholars get a lot of questions about including some crucial aspects like emotions, decision-making skills, analytical skills. When an AI is given the highest position in a system or work culture it 
should take care of works with utmost priority as humans do. The best example of this type of society is the automobile manufacturing sector. We see many robot arms assembling and fitting vehicles without any human intervention. This increases the production rate and efficiency. Tests like the crash test, Speed test, Safety checks can be done by the software itself. Here in this sector, we see a group of machines working together to give a car or a vehicle out of the factory. This is the best example of AI society ${ }^{[1]}$.

\section{Artificial Super Intelligence (ASI):}

Artificial intelligence (AI) involves the software-based simulation of human intellectual capabilities such as learning (the acquisition of information and rules for using the information), reasoning (using the rules to reach approximate or definite conclusions), and self-correction. As we all know about three phases of artificial intelligence namely ANI, AGI and ASI. ANI stands for Artificial Narrow Intelligence. In this phase, a set of instructions are executed only for the specified set of data. This phase is in the 1950s. In this phase, we see some studies about chatbots like ELIZA. We are currently in the phase of AGI. AGI stands for Artificial General Intelligence. We are experiencing benefits in this phase at present. This phase has many contributions like a virtual personal assistant, Cancer detection, Facial feature detection and many other areas. This is still in process. In this phase, only human intervention is slightly decreased in these autonomous applications. In the mere future, AI is going to be work without any human intervention ${ }^{[2]}$. This phase where AI creates its society and applications to help itself comes under the ASI phase. ASI stands for Artificial Super Intelligence. In this phase, machines think beyond human thinking capabilities. Machines can give efficient outputs in many fields like sports, medicine, art and many other crucial roles. When we see contributions in ASI phase self-driving cars, Virtual Assistants, VR applications, ATS tools these are some examples for ASI phase. In this phase many applications replace human roles. For example, take the human resources sector in Corporates this may be taken into control by software like SPSS and ATS ${ }^{[2]}$. The main aspect of researchers and scientists looking towards the ASI phase is ethics. Many questions arise regarding how AI can achieve ethics. Is it practically possible to evaluate good and evil on its own?

\section{FEW AREAS WHERE AI IS BEEN POPULAR:}

These days computers and machines became extremely powerful. We see many technical fields in the world like Automobile Industry, chatbots, Gaming, and even managerial fields like Marketing, finance, and the Banking sector. If we take marketing as our point of discussion for example if we take NETFLIX application, they provide predictive technology which suggests plenty of movies and their advertisements, web shows based on our age, country, previous search. We all heard about google lens. Google lens is an application where users can search for a product online by just taking snaps of the product. There is a vast advancement of Artificial Intelligence in the Banking sector also. HDFC bank launched a chatbot named EVA (Electronically Virtual Assistant"). This is a chatbot through which users can mention credit card frauds, Blocking their cards. EVA gives answers in less than 0.4 seconds which is a tremendous advancement of Artificial Intelligence. We see another upcoming area in artificial intelligence. Artificial Intelligence has been helping many of the farmers who need to work more smartly. There is a berlin based Agri-technical start-up "PEAT". They developed a smartphone application called "PLANTIX" through which soil nutrient deficiencies, plant viruses can be provided with just a snap from a smartphone. Through this, it compares from hygienic plants database and gives solutions for farmers. We 
also see DJI drones. They work on image recognition. Simply a farmer can plot his field on a map and sit aside with fitting pesticides to the drone. In stimulated all spraying tasks will be done by classifying the field into parts. In recent times we see attacking drones project on 26 January 2021 as part of Republic Day celebrations of India. Here a group of drones automatically detects fuel depots, enemy tanks, battle units using deep learning algorithms, and drop missiles and bullet pallets autonomously. This technology is designed and showcased by DRDO (Defence Research and Development Organization). Artificial Intelligence is helping NASA scientists to identify chemicals and particles on other planets. NASA designed a rover names "AEGIS" it operates in two modes one is the autonomous mode when it lost the link with earth when solar eclipse or night happens. AEGIS (Autonomous Exploration for Gathering Increased Science). NASA launched AEGIS in 2016 to MARS to collect samples of rocks and chemicals. It collects data through a pre-installed program called ChemCam. Nowadays Ai is vast in management fields like marketing, sales. There are many companies using AI to obtain good customer acquisition and service facilities. For example, if we take H\&M company they use a chatbot that handles customer queries and replies to them in a few seconds. These bots can help sales \& marketing people around them using customer experience and feedback obtained with the product.We see Artificial Intelligence in all these areas. These all areas are planned using human intervention marketing, finance, agriculture, AEGIS, chatbot these all applications are sorted out using a set of programs or tools. Now we will discuss Artificial Intelligence deciding on its own.

\section{FEW DISADVATAGES :}

We know well where there are advantages there more problems rises. When we take AI in the field of marketing and sales it cannot handle a typical client as a human does. It should make customers know about the product, its advantages and convince a customer to buy the product without any further deviation. But AI chatbot will give outputs in a trained manner with a limited number of options and information only. This may take lead to loss of customer from hand. As all studies say a customer is the first god to any company. This case cannot be neglected. We should keep in mind one thing that any programming logic or algorithm is designed by a human. So there is no guarantee for misuse of software or machine. For example, if we consider AI in Law Enforcement and Defence. In human scenario country soldiers of one country cross enemy lines to save or infiltrate a target that is miscellaneous. But this not a good ethical solution in the case of AI. When an order is given to a machine or robot it blindly obeys the rule. It may not verify whether it's good or evil. In this case, the complete system will be ruined. When we take a high crime rate city in a country. According to AI, it gives output in a previously trained way of conducting more patrols, increasing surveillance. But more protection and human presence are needed. This decision cannot be predicted by AI. When AI in some financial services like banks, ATM's. For example, when we take mortgage cases in banks. Here for supposing a bank uses a machine-learning algorithm to approve or reject applications. There is a chance of getting a lawsuit complaint against the bank. When a person files a case against a bank it is racially discriminating against people on the basis of race. This may become a huge headache to the bank in legal terms. On the other hand, AI is helping many in a positive light. For example, when we take auditors $\mathrm{Ai}$ is helping them in visualizing data in terms of decision tree algorithms and many else. So that a decent prediction can be achieved. As we discussed AI has a sea of opportunities to help with. But when ethics comes into the frame it might not be possible practically. For example, take the example of the self-driving autonomous car. Here a machine learning algorithm is trained with neural networks so that car understands its surroundings, road when to horn when to apply side indications. This autonomous vehicle is a great invention of the human. But without human intervention completely depending on the 
algorithm is completely unpredictable. This makes the situation worse. For example, a car has some technical failure brakes that are not functional in the car due to some malfunction. In this case, if an old woman and a child come in front. It may get into a confused state whom to save and whom to hit. If there is human handling the car he may reduce the gear or hit the divider. Of course, the car will be damaged or the person who is in the car may get severely wounded. But other pedestrians or persons or road won't get hit. In this case, AI is completely helpless in finding a solution. In medical terminology also there are many cases that AI is used is vast. But without human intervention, it may lead to severe liver damage in some cases. For example, take a surgeon he takes helps of the robotic arm he does a surgery based on the image he gets on screen. But in this case, if this arm gets failed by any case and it is stuck in the body of the patient. This will be very dangerous and anyone will become helpless.

\section{The Philosophy of AI with Ethics:}

We can state it as AI with Ethics or Robo Ethics. These terms define the morality of designing, constructing, and using robots. In this aspect, we can discuss AI becoming harm and AI becoming advantageous. We can define the study of human conduct, the concept of good and evil, right, wrong in a situation, Justice or Non-justice, moral assessments as ethics. It is very difficult to design a machine satisfying all concepts of ethics. If we discuss the good and evil concept in our day-to-day life. We cannot predict a particular person or system as good or evil. It completely depends on the perspective of viewing a situation. For example, there are two hackers one will be working for illegal sources like terrorist organizations and the other fellow will be working as the ethical hacker who works for government organizations. They both are doing the same job but the way sees their job role may change. An illegal hacker says he is working for real peace injustice that is not being achieved by the government. In his perspective, he thinks that he is working for a good cause. But from a societal point of view, he is a cyber threat. In human contexts, we already discussed human consciousness. Of course, arousal can be integrated by taking the help of neural networks in AI. We know human consciousness is nothing but aware of its state and actions. In brief, there are four levels in consciousness. These are described in an article called "space-time theory of consciousness". There it was specified that the levels will be Level 0, Level 1, level 2 , Level 3. In level 0 a group of plants with very small mobility almost negligible understand the state in surroundings by temperature. When we see in level 1 reptiles and insects they understand state through space including temperature, vision, smell. When we study level 2 i.e. mammals they will understand state through emotions and relationships to other species. Finally, when discussing humans they understand the state around them using senses, temperature, and all the above-mentioned features. So a machine should be designed in such a manner that it understands level 3 of consciousness. This can be achieved only through feedback loops and deep learning algorithms. There is a talk or discussion in society that AI may change or interrupt the behaviour of a workgroup in an organization. But in the same way, AI increases production rate, improves financial accountability, increases efficiency in the final output of the product. Many intellectuals argue that AI may fake results because of human intervention. We all know many of the Ai technologies work with machine learning algorithms. These algorithms work with datasets ${ }^{[3]}$. The datasets provided are the life of the system or project. When preparing or collecting a dataset user should be genuine. For example, take a college review software. Some colleges give fake results and their progress. As a result, when this data is fed into the algorithm results will appear in the way the dataset is designed. So here complete blame is the organization or person giving fake datasets. But people who experience the output of algorithms blame machines/software that it is giving fake results. A big question comes whether AI can judge or evaluate people on their 
behaviour as humans do. Several Computer vision algorithms like facial recognition, feature extraction, voice recognition are in fast phase in this area of detecting human personality. But it cannot predict human behaviour in future aspects whether he/she can succeed or not like astrologers. Many companies and countries often use AI surveillance systems to protect their work or cultural privacy and integrity. These services include secret service, firewall systems...etc ${ }^{[3]}$. In this digital sphere, all the systems may work with a single sheet called data. So, protecting data using encryption and other aspects is the main task of AI. AI can be ethically implemented when it is trained with real-time genuine datasets and commands. There is a scope of misusing AI as it is created by a human. So, it is practically impossible in some areas like police, the judge, the nurse, especially for elders. They need the utmost responsibility and care to manage some issues.

\section{MYTHS About AI:}

In many aspects, people think automation is the same as AI. But they are two different areas of discussion. In AI it is a machine or process that can think and do tasks through human inputs. Automation is nothing but completing a task form little to no human intervention. Many people argue that AI is complete technology and replaces all human job roles ${ }^{[4]}$. But when we discuss in real-time AI will provide many new opportunities. AI destroys all human jobs in the coming years is completely a myth. Whenever new technology evolves new jobs will be created to design and look after issues. Many people argue that consciousness is big trouble in AI, and it cannot be achieved. But in recent times we see humanoid robots like Sophia performing tasks like sensing and heat detection ${ }^{[4]}$. Some areas like judgement and law are practically impossible with AI at present. But it can change through providing genuine and exact datasets.

\section{AI Singularity:}

A singularity is an event that happens in the material world. In AI machines and robots are materials. We see a Sophia robot designed in Hong Kong. Sophia is called the most expressive humanoid robot. Sophia can answer questions from people in interviews using open source AI and Blockchain techniques. Singularity can be achieved by transitioning technologies from ANI phase to AGI phase. AI should be developed and concentrated more on neural networks. In many countries, factories and industries are adopting AI systems to make their task simpler. We saw an example of Facebook chatbots. In this experiment, two chatbots are made to chat with each other. Surprisingly after the chat, the chat description is completely in binary codes ${ }^{[5]}$. Scientists banned these robot-to-robot chats from this moment. Many researchers and scientists debate that way of exploring and improving intelligence could be more powerful by using an AI system ${ }^{[5]}$. Nick Bostrom states that AGI phase machines can bring humanity to extinction. These super-intelligent robots in the ASI phase can make things that are impossible to man. These machines can apply ideas on their own to improve work style from their past experiences. The problem when AI is this much developed is, they may kill other agents, impact them to change their behaviour or block them to attempt an interruption to the system. AI without human intervention is completely a risk. Instead of discussing these limitations, there are many advantages like disease detection, poverty and could help us in giving good predictions. The main benefit of AI is machines have no death. Their intelligence or working capabilities may increase from training a greater number of algorithms and datasets. We see many virtual assistants like Siri, Google, Alexa. These all are very helpful in recognizing voice commands and giving outputs. Tesla is the best example of machines in the ASI phase. Tesla cars are partially autonomous ${ }^{[5]}$. They work with human intervention. A Tesla car will not take 
complete authority on its own. The driver will be conscious and when any difficulty rises, the car automatically makes the driver acknowledged.

\section{Weaponization with AI:}

Artificial Intelligence machines/robots are designed by a human. The main issue rises here because a programmer can manipulate this system with his knowledge. When we see one example of this in the USA, the speech of Obama is faked using AI. They took a speech from his old recordings and trained the same voice to the system and converted his face emotions as for the speech. These were completely done by RNN algorithms. When issues like these come to light, they show how AI is very abusive. Many countries are now funding AI weaponry. The USA is funding an automatic CIWS project ${ }^{[6]}$. This automatic CIWS can destroy incoming missiles without human intervention. In hill countries like India and Pakistan artillery is a key weapon to fight fighter jets and a large group of soldiers for longrange targets. DRDO in its latest weapon expo showcased its autonomous artillery. Autonomous artillery is nothing but a heavy gun without human intervention. While firing an artillery gun it gives lots of sound and thrust which will be very difficult to bear. But using an autonomous gun need not affect our soldiers. When this technology is used in the country for fighting enemies it will be good ${ }^{[6]}$. But letting these types of technology into harmful hands make situations worse. Many atomic research and tests are being done with the help of AI. These make researchers and scientists test their weapons with very low life loss. In Republic Day celebrations of India 2021, Indian Army showcased its swarm of autonomous drones attacking enemy targets without human intervention. This technology is completely autonomous ${ }^{[6]}$. A drone identifies a tank or a fuel depot as it was trained with some images and when they come around of such targets, they use edge detection and inflate them. It completely works using computer vision algorithms.

\section{Decision Making with AI:}

In recent times AI is developed uniquely. We see autonomous self-driving cars from many automobile industries. They work on algorithms like image detection and object detection. They detect obstacles using high-definition cameras and stop the vehicle if they come across any. Many corporate companies use ATS and SPSS software to hire the right candidates ${ }^{[7]}$. They use AI algorithms to screen and select employees based on genuine talent. Many companies started a data-driven technique for operational decision making. In this process key feature is data. When we talk about the type of systems like AI-driven and Data-driven, AI-driven is completely autonomous and is updated by feedback loops. Datadriven algorithms are completely based on datasets provided. In data-supported decisionmaking big data plays a key role. The flow goes by summarized data succeeded by human judgement and final decision making. At present decision-making skills for AI are lacking. This is the key area stopping AI to achieve singularity ${ }^{[7]}$. For example, take a group of hunters. They use their sharp skills like listening and their vision to pretend risk and good taste. For suppose there is a bush in forest rustling. Humans do some acts or check whether it is a dangerous animal or a small animal that is rich with nutrients. If it is a predator, they might take some measures to protect their lives. But when we take the same example in AI robots, they cannot take a quick decision when a predator comes. It results in complete failure of the system. Cognitive thinking abilities of AI is also a major factor for AI to achieve singularity. They can be achieved by continuous evolution and training of the latest data to the system. Data is not only enough to insulate us from cognitive bias. A human can predict by seeing a chart or group of items whether it is genuine or not. But an AI or a machine cannot predict based on the data. It needs some general knowledge of whether the data is 
genuine or not. So, decision-making skills in AI should be developed in the same way humans do to achieve singularity

\section{Viewpoint:}

We all are living in an upgrading world. Every day is new. The day with new technology rising. In this world with a lot of population and employees. It is very difficult to handle so many roles manually. So using autonomous systems is much recommendable. Of course, there are some disadvantages with AI when we consider ethics. But keeping aside these limitations there is a large number of advantages. Controlled AI can be very useful in all areas. Maximum machine learning algorithms make tasks autonomous. But giving complete control to machines without any human intervention is way too dangerous. In recent times consciousness is slightly in the entry phase in the field of AI. We are in the phase of AGI so it may get some of the features in consciousness with the training of data.

Even an AI system is trained with consciousness smell and taste cannot be predicted. As smell is taken care of by the human nose and smell donot have sensors to find this area is impossible. And same issue rises with taste also if you take a paneer pizza it cant predict taste like how much spicer it is. But imagine the situation of satirical dialogue rolled on you. Humans will face this situation in two ways one will be giving a polite reply or giving a satirical reply in the same manner. But this is practically impossible in the case of AI. In this case, just think how AI can guess it is a satire. Such decisions or actions that include emotions may become a drawback in this concept. When we talk about ethics it is very difficult for a system to train all goods and evil in society and in real-time. So predicting or giving a statement in such a situation is a bit difficult. Even now AI is into detecting the face of individuals and predicting personalities based on facial features. It cannot predict a person's behavior based on continuous action. It predicts the personality of the person based on previously defined data only. So AI without human intervention is a bit difficult to describe.

\section{Conclusion:}

As discussed key areas of AI when included with consciousness and ethics. In some cases, this might not be achieved. In some areas like auditing, autonomous robotic arm in manufacturing, Packaging, classifying different products in stores...etc AI is highly suggested. In all these areas a programming logic or previously trained data is given as reference so a maximum of the applications no needs human intervention. In space applications like rovers and launchers, we see many types of research being done on MARS using CURIOSITY rover. This rover is completely working on AI without any human intervention. But in some risky areas like autonomous vehicle driving, Medicine human intervention is a must as there is a chance of malfunction this may lead to vulnerable damage that may cost lives. Consciousness can be achieved in some areas only like arousal, sense, feel. But when we take an example of smell and taste result may vary. So we cannot predict that AI is complete consciousness. When AI is not completely conscious it is very difficult to predict real-time scenarios. When we talk about ethics this is highly impossible as we discussed police and law enforcement AI might get confused because criminals might mislead the system by knowing about it. So there is a risk of the wrong person being caught. When we talk about the programming part or algorithm part of AI we should keep in mind that any logic or piece of code is done by a human. When we talk about cognitive abilities motor skills, visual perception, Linguistic skills Ai can be trained using neural networks. A machine can make movements with human commands. It can make things works based on 
the environment. AI might face some problems in decision making and critical thinking. This should be kept in mind when we depend on AI systems. In some cases, decisions may change because of bias done by programmers or companies. So there is a chance of bias situation. This may lead to complete failure of the system. So AI without human intervention is unpredictable.

\section{References:}

[1] The impact of AI on business, society, and everyday life. (2021). Retrieved 17 February 2021, from

https://cloud.withgoogle.com/build/data-analytics/impact-ai-business-society-everyday-life/

[2] A new dawn. (2021). Retrieved 17 February 2021, from https://www.ubs.com/microsites/artificial-intelligence/en/newdawn.html\#: : :text=AI\% 20is\% 20divided\%

[3] Ethics of Artificial Intelligence and Robotics (Stanford Encyclopaedia of Philosophy) . (2021). Retrieved 17 February 2021, from https://plato.stanford.edu/entries/ethics-ai/

[4] Marr, B. (2021). 5 Myths About Artificial Intelligence (AI) You Must Stop Believing. $\begin{array}{llll}\text { Retrieved } & \text { February } & \text { 2021, from }\end{array}$ https://www.forbes.com/sites/bernardmarr/2017/10/03/5-myths-about-artificialintelligence-ai-you-must-stop-believing/?sh=297580352739

[5] The "father of artificial intelligence" says singularity is 30 years away. (2021). Retrieved 17 February 2021, from https://futurism.com/father-artificial-intelligence-singularitydecades-away

[6] Pandya, J. (2021). The Weaponization Of Artificial Intelligence. Retrieved 17 February 2021, from_https://www.forbes.com/sites/cognitiveworld/2019/01/14/the-weaponizationof-artificial-intelligence/?sh=5f90a5736867

[7] How AI Can Transform the Decision-Making Process | Ayehu. (2021). Retrieved 17 February 2021, from https://ayehu.com/ai-for-decision-making/ 